\title{
Contrast sensitivity to angular frequency stimuli is higher than that for sinewave gratings in the respective middle range
}

M.L.B. Simas,

N.A. Santos

and F.A. Thiers

\author{
Laboratório de Percepção Visual, LabVis-UFPE, \\ Departamento de Psicologia, Universidade Federal de Pernambuco, \\ 50670-901 Recife, PE, Brasil
}

\author{
Correspondence \\ M.L.B. Simas \\ Laboratório de Percepção Visual \\ LabVis-UFPE \\ Departamento de Psicologia, UFPE \\ 50670-901 Recife, PE \\ Brasil \\ E-mail: mlbslabvis@npd.ufpe.br or \\ mlbslabv@elogica.com.br \\ Research supported by CNPq \\ (No. 31.1047/84.0) and FINEP \\ (No. 43.88.0234-00/Projeto 2).
}

Received November 4, 1996 Accepted February 18, 1997

\begin{abstract}
This study compares contrast thresholds for sinewave gratings, or spatial frequencies (1/CSF) with contrast thresholds for angular frequencies (1/aCSF) and for radial frequencies, or $\mathrm{J}_{0}$ targets $(1 / \mathrm{rCSF})$. Observers had to differentiate between one of these frequency stimuli and a stimulus at mean luminance within a forced-choice procedure. All measurements were made with the same equipment, methods and subjects. Our results show higher sensitivity to, or lower thresholds for, angular frequencies when compared to either sinewave gratings or $\mathrm{J}_{0}$ targets. Contrast values in arbitrary units, in the lower threshold range for angular frequencies, were about half those required to differentiate sinewave gratings from mean luminance in its most sensitive range.
\end{abstract}

Since their initial use in vision studies in 1948 (1,2), sinewave gratings have aroused intense interest on the part of researchers due to their high potential to characterize responses of the visual system as a whole or to characterize individual cell unit responses. Many comparisons of contrast thresholds to sinewave gratings for the human visual system have been made in the literature with the use of psychophysics procedures. As examples, we can cite comparisons among contrast thresholds for squarewave gratings, lines, edges and borders (3-7). Comparisons have also been made with human contrast thresholds for $\mathrm{J}_{0}$ targets $(8,9)$. In the present text, $\mathrm{J}_{0}$ targets are synonymous with radial frequencies. We know of no comparisons made with thresholds for angular frequencies $(10,11)$, the orthogonal component of radial frequencies when a polar coordinate system is adopted (12).

In the present study we measured contrast thresholds for sinewave gratings (spatial frequencies), for $\mathrm{J}_{0}$ targets (radial frequencies) and for angular frequencies (as previously defined in Refs. 10-12) using the same equipment, method and procedures as well as subjects. We define angular frequency as circular stimuli whose contrast are modulated by sinewave functions varying with angles within 360 degrees. Due to this 360degree limitation, we made $n$, i.e., the number of cycles, an integer.

The inverse of the curve of contrast thresh- 
olds for sinewave gratings, or spatial frequencies, for the human visual system is also known as contrast sensitivity function (CSF), and sometimes identified as modulation transfer function (MTF) (13). Thus, we define the responses to sinewave gratings as CSF, and the same curves for both radial or angular frequencies as rCSF and aCSF, respectively.

The curves were measured for eight observers, AMB, BMD, FAT, JTF, LFO, NAS, YEQ and WGC, with normal or optically corrected visual acuity. Not all observers had measurements made for all three curves.

The patterns were generated in 256 gray levels on a high-contrast resolution monitor (Sony BVM-1910) with interlaced RGB input and interfaced to a DT-2853 frame grabber controlled by an IBM compatible microcomputer. Contrast could be set digitally by the computer. All experiments were run at a mean luminance of $8.2 \mathrm{~cd} / \mathrm{m}^{2}$. Maximum and minimum luminances were 10.3 and 6.2 $\mathrm{cd} / \mathrm{m}^{2}$, respectively. Contrast was assumed to vary linearly with digital setting. A neutral gray board was used for fixation between trials.

Estimates of the CSF and rCSF were obtained at spatial frequencies of $0.2,0.3$, $0.5,0.8,1,2,3,4,5,6,9$ and 12 cycles per degree (cpd). Those for the aCSF were obtained with point estimates at angular frequencies of 1, 2, 3, 4, 6, 9, 13, 16, 24, 32, 47, 64 , and 96 cycles per 360 degrees. All stimuli subtended 7.25 degrees of visual angle viewed from a distance of $1.50 \mathrm{~m}$. The procedure involved independent measurements of the three curves with at least the same 2 observers for all curves.

A total of 9 curves containing 12 points were measured for the CSF (BMD:2, WGC:2, JTF:1, NAS:2, FAT:2) during 108 experimental sessions lasting about 15-25 min each. Similarly, 13 curves of 12 points were measured for the rCSF (BMD:1, WGC:2, JTF:2, NAS:2, FAT:2, AMB:2, LFO:2), yielding a total of about 156 sessions, and 9 other curves of 13 points were measured for the
aCSF (LFO:2, AMB:2, NAS:2, FAT:2, YEQ:1), for a total of 117 additional sessions.

When two measurements were made by the same observers for the same condition they were never made on the same day. The order was randomly assigned for each observer. The method used has been described in Refs. 10-12. In each forced-choice trial sequence, one of two observation intervals (each lasting $2 \mathrm{~s}$ in contrast to $10 \mathrm{~s}$ in Ref. 12) randomly contained the test stimulus while the other contained the mean-luminance-only stimulus. The blank intervals between stimuli, ISI, and between trials, ITI, lasted 1 and $3 \mathrm{~s}$, respectively. Following the presentation of the second stimulus interval, observers were required to identify the interval containing the test frequency stimulus. The computer would pause until an answer was entered and a beep would indicate a correct choice. The fixation point was at the center of the stimulus and, to avoid possible brightness aftereffects during ITI, observers fixed their gaze on a mark on the gray board while waiting for another beep indicating when to look at the screen. Observers were also told to restrain eye movements during each 2 -s stimulus presentation as much as possible, all measurements being made binocularly. Contrast modulation of the test stimulus could be varied in steps of $2 / 256$, i.e., $0.8 \%$, and had an initial setting of $8-11 \%$. The criterion was three consecutive correct trials to decrease contrast by one step, and a single incorrect trial to increase it by one step. This criterion yielded a frequency of seeing level equal to $0.51 / 3$, or $79 \%$ (14). In case either the upper or lower limits of contrast were reached (i.e., either 0.008 or 0.992), the computer would repeat that contrast level again and again until the response of the subject reversed direction. Two to 4 conditions were run per day with each observer, always with a 10-15-min interval between conditions.

In each experimental session, a total of 
10 maximum-minimum pairs were obtained per observer. Thus, each point on a curve estimate is the average of 20 contrast thresholds per subject. If 13 such curves are determined, each point is the average of 260 contrast threshold determinations.

Figure 1 shows examples of test stimuli used for the measurements. We can see angular frequencies for 24 cycles (top left), 16 cycles (top right), 13 cycles (center left), and 2 cycles (center right) as well as a sinewave grating for $4 \mathrm{cpd}$ (bottom left, when viewed at a $1.5-\mathrm{m}$ distance) and a blank at mean luminance (bottom right), the latter being present in all experimental conditions.

Figure 2 shows the results for the three measured curves, i.e., the CSF, rCSF and aCSF. Grandmeans of contrast thresholds across subjects are shown for spatial, radial and angular frequencies, respectively.

We make a note that, by definition, angular frequencies are adimensional and only integers, and that, therefore, though the abscissa is equivalent for both spatial and radial frequencies, it is not equivalent to angular frequencies. On the other hand, contrast thresholds are plotted on the ordinate in the same scale of arbitrary units since all measurements were made using the same equipment and computer-controlled procedure. Error bars are corrected by the Student $t$-test distribution to be at the $99 \%$-confidence interval.

Minimum contrast threshold levels occurred at 2.0-3.0 cpd, i.e., 0.0329 and 0.0338, or $3.29 \%$ and $3.38 \%$, for the $1 / \mathrm{CSF}$. In comparison, minimum contrast threshold levels for the $1 / \mathrm{rCSF}$ occurred about $0.8-1.0 \mathrm{cpd}$, i.e., 0.2082 and 0.2046 , or $20.8 \%$ and $20.4 \%$. The latter contrast levels were about 6-fold higher than those for the 1/CSF. In contrast, minimum contrast thresholds for the $1 / \mathrm{aCSF}$ were found to vary around a much larger plateau, i.e., 13, 16, 24 and 32 cycles. The values for these thresholds were about $0.0210,0.0215,0.0228$ and 0.0201 , or $2.1 \%$, $2.2 \%, 2.3 \%$ and $2.0 \%$, i.e., about 1.6-fold

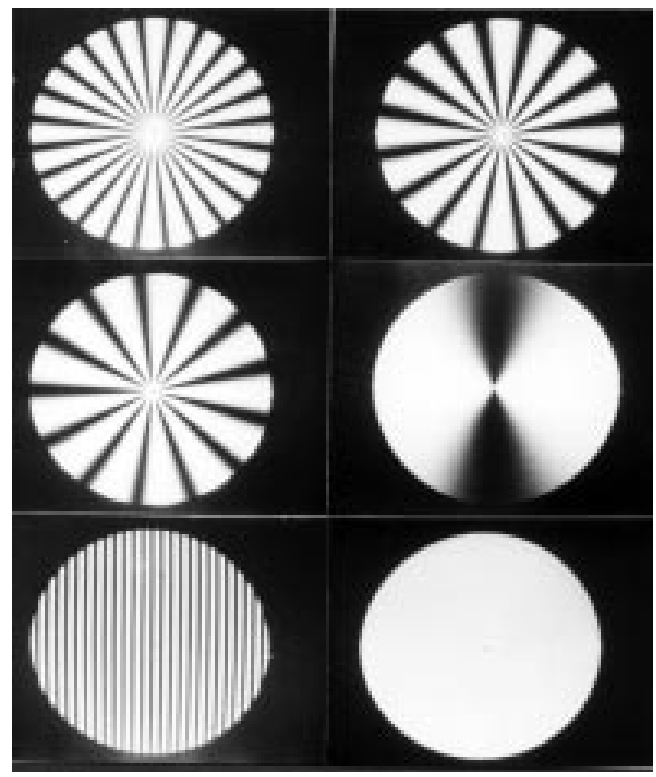

lower than the levels found for the $1 / \mathrm{CSF}$ in its most sensitive range.

Although we could see that these results were consistent with an independent probability summation effect among channels tuned to different spatial orientations, this may not be an explanation for higher aCSF sensitivity since cells tuned to one orientation tend to be inhibited by non-preferred orientations $(15,16)$. We interpret our results as being consistent with the idea of conceiving the visual system as decomposing the

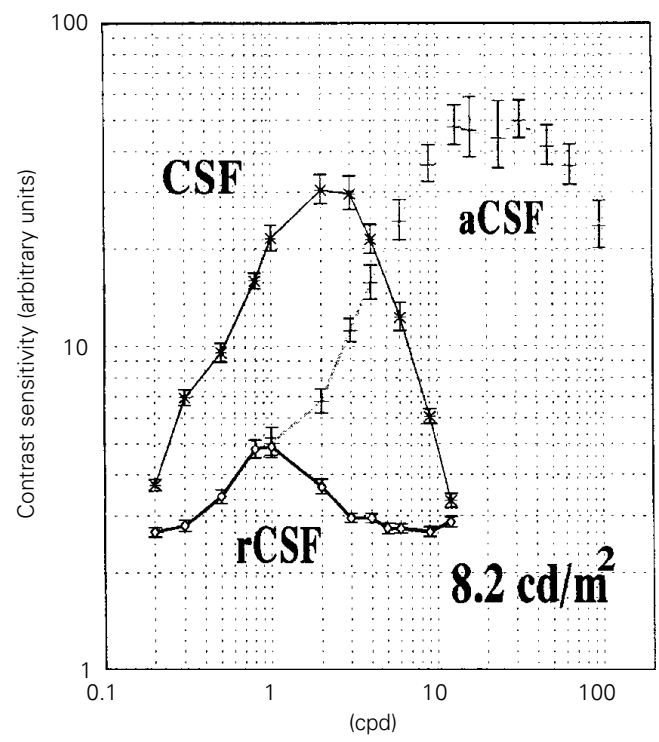

Figure 1 - Examples of test stimuli used for measurements. Angular frequencies for 24 cycles are seen at top left, for 16 cycles at top right, for 13 cycles at center left, and for 2 cycles at center right. An example of sinewave grating at 4 cpd is at bottom left (the spatial frequency is correct only as viewed at $1.5-\mathrm{m}$ distance in the actual experiment). A blank at mean luminance is seen at bottom right and was present in all experimental conditions. Observe that no Jo targets or radial frequencies are shown.

Figure 2 - Estimated CSF ( $N=$ 9), $\operatorname{rCSF}(N=13)$ and $\operatorname{aCSF}(N=$ 9). Grandmean of contrast threshold reciprocals across subjects are shown for spatial, radial and angular frequencies, respectively. Note that, by definition, angular frequencies are adimensional and only integers, and that, therefore, though the abscissa is equivalent for both spatial and radial frequencies, it is not equivalent to angular frequencies. Contrast threshold reciprocals are plotted on the ordinate in the same scale of arbitrary units since all measurements were made using the same equipment and computercontrolled procedure. Error bars are corrected to indicate the 99\%-confidence interval by the Student $t$-test distribution. 
incoming spatial visual information in terms of polar coordinates. In this idea, the processing of a lower level contrast range involves mainly a default sampling for angular frequency decomposition by the visual system, encompassing peripheral and foveal vision. In contrast, the processing of higher contrast ranges would involve mainly foveal vision which seems to be better equipped for detecting high-band radial frequencies at central vision. As we see it, sinewave gratings could be processed as a by-product of the visual system sampling whereas angular fre- quencies would be detected mainly at higher levels of visual neuronal processing, i.e., at levels where the visual system could be integrating spatial frequency information either per hemisphere or per portion of visual quadrants. On this basis, we could also argue that sensitivity to spatial frequencies represented by sinewave gratings may be a particular case of visual sampling. Recent results obtained in physiological studies show evidence consistent with some aspects of these hypotheses $(17,18)$.

\section{References}

1. Selwyn EWH (1948). The photographic and visual resolving power of lenses. Photographic Journal, 88B: 6-12.

2. Schade $\mathrm{OH}$ (1948). Electro-optical characteristics of television systems. 1. Characteristics of vision and visual systems. $R C A$ Review, 9: 5-37.

3. DePalma JJ \& Lowry EM (1962). Sinewave response of the visual system. II. Sine-wave and square-wave sensitivity. Journal of the Optical Society of America, 52: 328-335.

4. Lowry EM \& DePalma JJ (1961). Sinewave response of the visual system. I. The Mach phenomenon. Journal of the Optical Society of America, 51: 740-746.

5. Campbell FW \& Robson JG (1968). Application of Fourier analysis to the visibility of gratings. Journal of Physiology, 197: 551-566.

6. Kulikowski JJ \& King-Smith PE (1973). Spatial arrangement of line, edge, and grating detectors revealed by sub-threshold summation. Vision Research, 13: 1455-1478.
7. King-Smith PE \& Kulikowski JJ (1975). The detection of gratings by independent activation of line detectors. Journal of Physiology, 247: 237-271

8. Kelly DH \& Magnuski HS (1975). Pattern detection and the two-dimensional Fourier transform: circular targets. Vision Research, 15: 911-915.

9. Kelly DH (1982). Motion and vision. IV. Isotropic and anisotropic spatial responses. Journal of the Optical Society of America, 72: 432-439.

10. Simas MLB (1985). Linearity and domain invariance in the visual system. Doctoral thesis, University Microfilms International, Publication No. 8617940, 156-198.

11. Simas MLB, Frutuoso JT \& Vieira FM (1992). Inhibitory side bands in multiple angular frequency filters in the human visual system. Brazilian Journal of Medical and Biological Research, 25: 919-923.

12. Simas MLB \& Dodwell PC (1990). Angular frequency filtering: a basis for pattern decomposition. Spatial Vision, 5: 59-74.

13. Cornsweet TN (1970). Visual Perception. Academic Press, New York, 311-364.
14. Wetherill GB \& Levitt $H$ (1965). Sequential estimation of points on a psychometric function. British Journal of Mathematical and Statistical Psychology, 18: 1-10.

15. Hubel DH \& Wiesel TN (1962). Receptive fields, binocular interaction and functional architecture in the cat's visual cortex. Journal of Physiology, 160: 106-154.

16. Hubel DH \& Wiesel TN (1968). Receptive fields and functional architecture of monkey striate cortex. Journal of Physiology, 195: 215-243.

17. Gallant JL, Braun J \& Van Essen DC (1993). Selectivity for polar, hyperbolic, and Cartesian gratings in macaque visual cortex. Science, 259: 100-103.

18. Gallant JL, Connor CE, Rakshit S, Lewis JW \& Van Essen DC (1996). Neural responses to Polar, Hyperbolic, and Cartesian gratings in area $\mathrm{V} 4$ of the macaque monkey. Journal of Neurophysiology, 76: 2718-2739. 